\title{
FUNCTION
}

\section{The Role of Still-Frame Parametric Imaging in Magnetic Resonance Assessment of Left Ventricular Wall Motion by Non-cardiologists}

\author{
Enrico G. Caiani, Ph.D., ${ }^{1,2}$ Eran Toledo, Ph.D., ${ }^{1}$ \\ Peter MacEneaney, M.D., ${ }^{1}$ Keith A. Collins, M.S., ${ }^{1}$ \\ Roberto M. Lang, M.D., ${ }^{1}$ and Victor Mor-Avi, Ph.D. ${ }^{1, *}$ \\ ${ }^{1}$ Noninvasive Cardiac Imaging Laboratory, University of Chicago Medical Center, \\ Chicago, Illinois, USA \\ ${ }^{2}$ Dipartimento di Bioingegneria, Politecnico di Milano, Milan, Italy
}

\begin{abstract}
Background. Cardiac magnetic resonance (MR) images are often reviewed by noncardiologists who are not trained in the interpretation of regional left ventricular (LV) function. We hypothesized that the use of still-frame parametric MR images of wall motion could aid in the assessment of regional LV function. Methods. Dynamic, electrocardiogram-gated, steady-state free precession (FIESTA) short-axis images were obtained in 6 to 10 slices in 18 consecutive patients. Each loop was used to automatically generate a still-frame image, in which each pixel is assigned a value equal to the amplitude of cyclic variation in local intensity, resulting in higher intensity in pixels that change between blood and tissue during the cardiac cycle. The dynamic images were reviewed by an expert cardiologist who provided gold standard grades for regional wall motion and by four radiologists. Then the radiologists reviewed and graded the same MR images in combination with parametric images. Grades assigned to each segment in the two sessions were compared with the gold standard. Results. According to expert interpretation, 6 patients had normal wall motion, and 12 had wall motion abnormalities. Parametric images showed a bright band in the area spanned by endocardial motion, with reduced brightness and thickness in areas of hypokinesis. The agreement between the radiologists' grades and the gold standard significantly improved by adding parametric images (from $77 \%$ to $81 \%$ ), which also resulted in reduced interobserver variability (from $52 \%$ to $33 \%$ ).
\end{abstract}

*Correspondence: Victor Mor-Avi, Ph.D., Noninvasive Cardiac Imaging Laboratory, University of Chicago Medical Center, MC5084, 5841 S. Maryland Ave., Chicago, IL 60637, USA; Fax: (773) 702-1034; E-mail: vmoravi@medicine.bsd.uchicago.edu. 
Conclusions. Still-frame parametric images aid in the assessment of regional wall motion by non-cardiologists who are required to interpret cardiac images.

Key Words: Left ventricular function; Endocardial motion; Magnetic resonance imaging; Computer analysis.

\section{INTRODUCTION}

Although techniques for objective, quantitative evaluation of regional left ventricular (LV) function from magnetic resonance (MR) images are commercially available, these techniques rely on tedious and timeconsuming, manual or semiautomated tracing of endocardial and epicardial boundaries. As a result, routine clinical evaluation of regional LV wall motion is still mostly based on visual interpretation of dynamic twodimensional images of the heart. This methodology relies on the reader's ability to integrate spatial and temporal information on endocardial excursion and wall thickening and, thus, is subjective and experience dependent, similar to the echocardiographic assessment of LV wall motion (Cohen et al., 1998; Picano et al., 1991; Popp et al., 1998), despite the superior quality of MR images.

As an attempt to overcome these limitations, we recently developed a fully automated technique that provides a simplified display that integrates spatial and temporal information on LV wall motion by creating still-frame parametric images of wall motion (Caiani et al., 2002.) We tested this approach on echocardiographic images and found that the addition of parametric images to complement visual interpretation of dynamic images improved the accuracy of the evaluation of LV wall motion by inexperienced reviewers (Caiani et al., 2002.) We hypothesized that still-frame parametric images obtained from cardiac MR cine loops could also aid in the assessment of LV wall motion. Accordingly, the aim of this study was to determine whether the use of parametric images in combination with dynamic MR images could improve the accuracy of visual interpretation of regional LV wall motion by non-cardiologists who have not completed dedicated and extensive training in the evaluation of regional wall motion.

\section{METHODS}

Images obtained in 18 consecutive patients (10 males, 8 females; aged $55 \pm 15$ years), referred for clinically indicated cardiac MR examinations were enrolled in this study. The only exclusion criteria were the known contraindications for magnetic resonance imaging. Imaging was performed by using a 1.5-T scanner (General Electric, Milwaukee, WI). Steadystate free precession (FIESTA) cine loops were obtained during 12-sec breath-holds with a temporal resolution of 20 frames per cardiac cycle, in 6-10 short-axis 9-mm slices from the atrioventricular ring to the apex. Digital images were analyzed off-line with use of custom-designed software.

For each cine loop, images were first cropped to isolate the left ventricle. For each pixel, values were extracted throughout the cardiac cycle to create time series of videointensity (Fig. 1). Each time series was fitted into a sinusoidal function: $y(t)=A_{1} \cdot \sin \left(\omega t+\varphi_{1}\right)+$ $A_{2} \cdot \sin \left(2 \omega t+\varphi_{2}\right)$ by using the standard least squares analysis. The period of this function, $T=(2 \pi \omega)^{-1}$, was determined from the electrocardiogram (ECG) $R-R$ interval. The amplitude of the first and second harmonic of the best-fit curve, $A_{1}$ and $A_{2}$, were calculated, and their sum was displayed in each pixel, resulting in a stillframe parametric image of the amplitude of oscillation in local pixel intensity encoded in gray scale (Fig. 1). Phase information contained in the values of $\varphi_{1}$ and $\varphi_{2}$ was discarded. This algorithm was implemented by using commercial image analysis software (Matlab Image Processing Toolbox, MathWorks, Inc., Natick, MA). The process of creating parametric images was fully automated and took less than 10 seconds per cine loop on a $1.3-\mathrm{GHz}$ personal computer.

Dynamic cardiac MR images were reviewed by an experienced cardiologist, blinded to the patient's identity and diagnosis, who classified wall motion in each segment in each slice as either normal or abnormal. This review was based on a standard segmentation scheme used in echocardiography for grading regional LV wall motion in the short-axis view (Schiller et al., 1989) and defined the gold standard interpretation of regional wall motion. Subsequently, the dynamic cardiac MR images were independently reviewed, and regional wall motion was graded as normal or abnormal by four radiologists with different levels of experience in the interpretation of cardiac images, ranging from 10 to 100 studies. During the reading, the radiologists were unaware of the gold standard as well as the other observers' interpretations. 

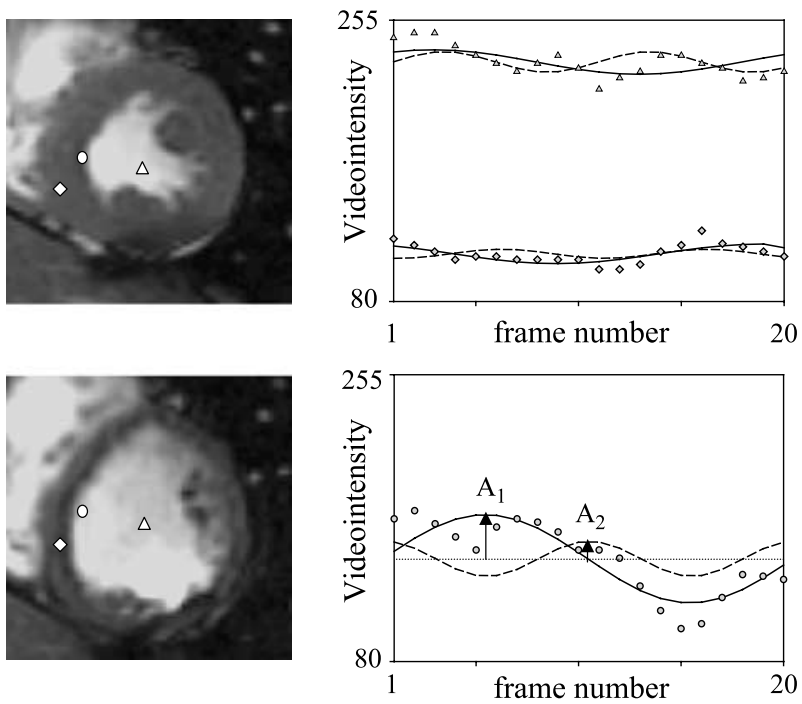

Figure 1. The principle of parametric imaging of left ventricular wall motion. The amplitude of oscillations in pixel intensity in a cine loop of MR images is dependent on the anatomic location of each pixel. As an example, three pixels at fixed positions are marked on the end-systolic (top left) and end-diastolic (bottom left) MR images with three different symbols: a triangle, a diamond, and a circle. Pixels that remain in the myocardium or in the ventricular cavity throughout the cardiac cycle (diamond and triangle, respectively) are not expected to change beyond image noise (top right). In contrast, the intensity in the pixel represented by the circle changes considerably as the endocardium moves in and out (bottom right). The sum of the amplitudes of the first two harmonics $\left(A_{1}\right.$ and $\left.A_{2}\right)$ of the best-fit function, which is the parameter displayed in the parametric images, thus can differentiate between these classes of pixels and, therefore, depict the extent of endocardial motion.

In a separate session, the radiologists were informed of the nature of the parametric images and the information displayed there; they were shown four examples of cases that were not included in the study. Then the classification of segmental wall motion was repeated by the same non-cardiologist readers who reviewed the dynamic loops in combination with the still-frame parametric images. The order of cases presented in the two sessions was randomized.

In each session, the individual non-cardiologist interpretations were compared to the gold standard by counting the segments where concordant (true positive and negative) as well as discordant (false positive and negative) readings were made. Segment counts were used to calculate the sensitivity, specificity, and accuracy of the non-cardiologist interpretations. In ad- dition, individual readings were analyzed to obtain the interobserver variability, which was defined as the percentage of segments in which the interpretation of at least one of the readers was different from that of the others.

The improvement in sensitivity, specificity, accuracy, and interobserver variability achieved by adding parametric images was tested by using the Z-test with Yates correction and considered significant for $p<0.05$. These analyses used sensitivity, specificity, accuracy, and variability values as sample proportions and the relevant number of segments as sample sizes.

Separate analyses were performed on data obtained in a subset of patients with regional wall motion abnormalities, as defined by the gold standard readings. Sensitivity, specificity, accuracy, and interobserver variability, as well as statistical analyses, were recalculated to assess the benefit of adding parametric images in this subset of patients with abnormal wall motion.

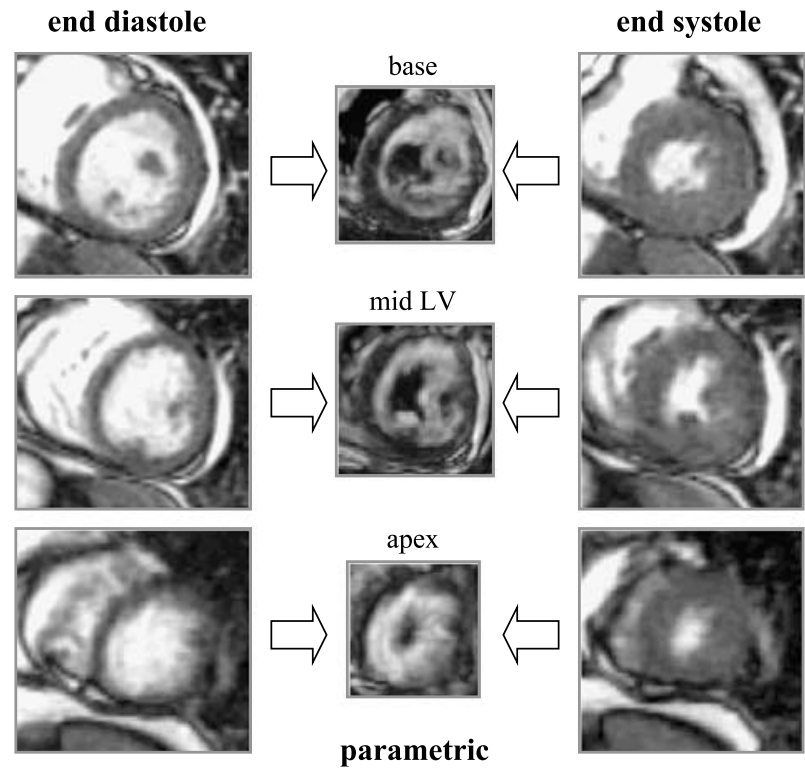

Figure 2. Example of end-diastolic (left column) and endsystolic (right column) short-axis MR images obtained at three different levels of the left ventricle in a patient with normal wall motion. The still-frame parametric images of wall motion (middle column) show a bright band, the inner and outer boundaries of which correspond to the end-systolic and enddiastolic positions of the endocardium. Thus, the thickness of the bright band reflects the extent of endocardial motion, which in this patient is fairly uniform at the midventricular level and near the apex, and appears thinner in the septal area in the basal slice near the aortic outflow tract. 

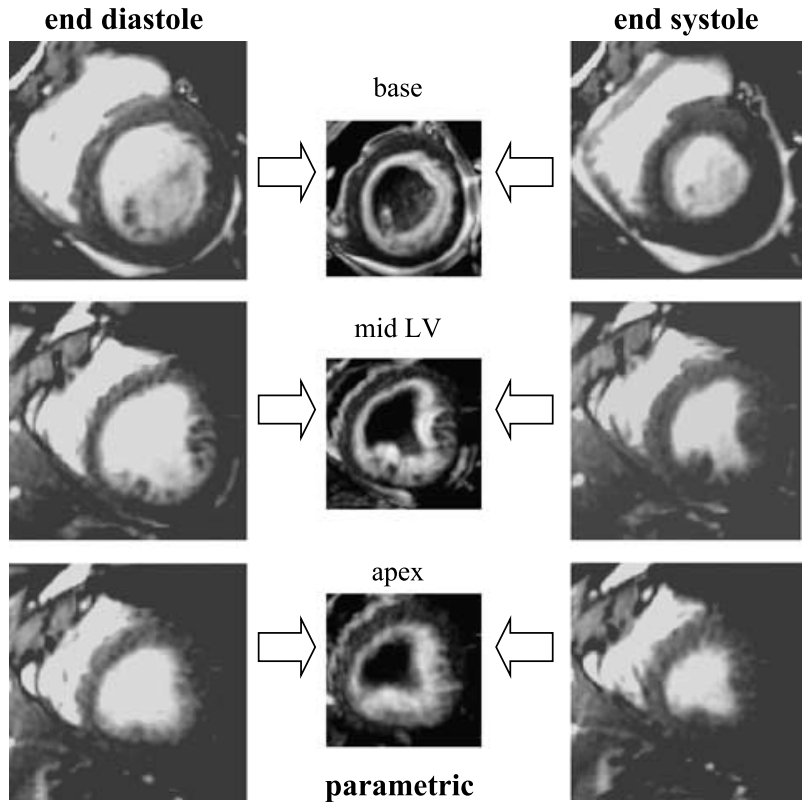

Figure 3. Example of end-diastolic (left column) and endsystolic (right column) short-axis MR images obtained at three different levels of the left ventricle in a patient with a resting wall motion abnormality in the interventricular septum secondary to $95 \%$ stenosis of the left anterior descending coronary artery. Note the reduced myocardial thickening (left and right columns) concomitant with the thinning of the bright band (middle column) in the septal area at all ventricular levels.

\section{RESULTS}

According to the gold standard expert interpretation, of the 18 patients enrolled in this study, 6 had normal wall motion, 10 had regional wall motion abnormalities, and 2 had global hypokinesis secondary to dilated cardiomyopathy. Of 768 segments examined (128 slices, 6 segments per slice), 541 were classified as normal and 227 as abnormal. Basal segments that contained the aortic outflow tract during at least part of the cardiac cycle were excluded from analysis.

Figure 2 shows an example of end-systolic (right column) and end-diastolic (left column) cardiac MR images obtained in different slices in a patient who, according to the gold standard interpretation, had normal wall motion. The resultant parametric images (middle column) showed a continuous bright band surrounding the LV cavity, reflecting normal wall motion, because the pixel intensity in the area spanned by the endocardial motion changes from high values, typical of the LV cavity in diastole, to lower values, typical of myocardial tissue in systole. Overall, we found that in patients with normal wall motion, the thickness of the bright band is fairly uniform in all short-axis slices, with the exception of the basal slices, where it was visibly thinner in the anteroseptal and septal segments adjacent to the LV outflow tract, which are known to be less dynamic.

Parametric images obtained in patients with regional wall motion abnormalities showed thinned bright bands in segments classified by the gold standard as abnormal. Figure 3 shows an example of MR images along with parametric images of wall motion obtained in one of these patients. Examination of end-systolic and end-diastolic MR images (Fig. 3, right and left columns, respectively) reveals reduced thickening of the septal wall, and the parametric image shows a thinned bright band reflecting hypokinesis in that area. Figure 4 shows an example of images obtained in a patient with dilated cardiomyopathy, where the overall thickness of the bright band is minimal, reflecting global hypokinesis.

Table 1 presents the sensitivity, specificity, accuracy, and interobserver variability data calculated for the non-cardiologist readings of the dynamic MR images, with and without parametric images. Adding parametric images to the dynamic cine loops resulted
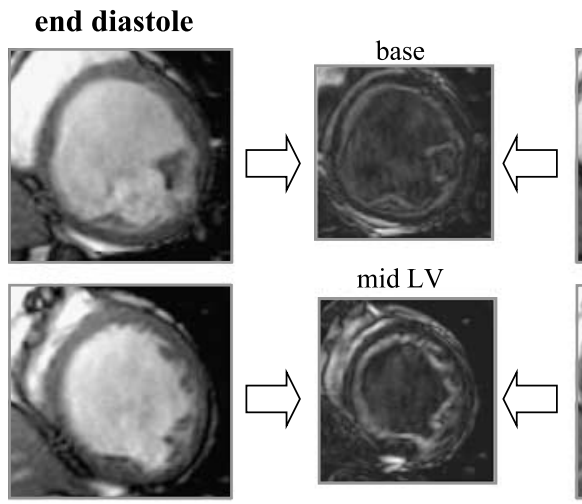

end systole

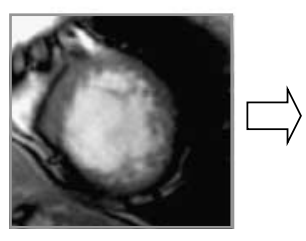

mid LV
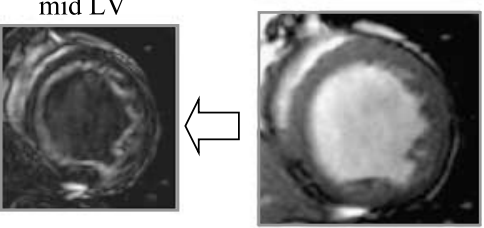

apex
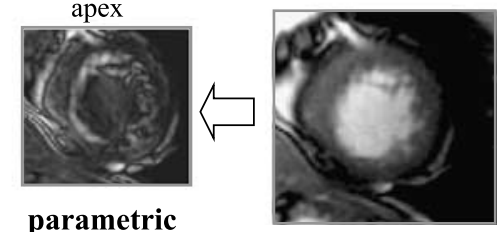

parametric

Figure 4. Example of end-diastolic (left column) and endsystolic (right column) short-axis MR images obtained at three different levels of the left ventricle in a patient with global hypokinesis secondary to dilated cardiomyopathy. Note the global reduction in myocardial thickening (left and right columns) and the reduced thickness of the bright band (middle column) in all myocardial segments. 
Table 1. Interpretation of left ventricular wall motion with and without parametric images.

\begin{tabular}{lcccc}
\hline & Sensitivity $(\%)$ & Specificity $(\%)$ & Accuracy $(\%)$ & Interobserver variability $(\%)$ \\
\hline Dynamic & $80(74)$ & $76(70)$ & $77(71)$ & $52(58)$ \\
Dynamic + parametric & $83^{*}(77)$ & $80^{*}\left(75^{*}\right)$ & $81^{*}\left(76^{*}\right)$ & $33^{*}\left(42^{*}\right)$ \\
\hline
\end{tabular}

Sensitivity, specificity, and accuracy of the interpretation of regional LV wall motion by four non-cardiologists calculated against the gold standard expert interpretation. Interobserver variability was calculated as percent of segments in which the interpretation of at least one of the four non-expert readers was different from those of the others. The value of still-frame parametric images was judged by comparing the interpretations made by the non-cardiologists' based on dynamic images alone and those based on the combination of dynamic and parametric images $(* p<0.05)$. Results are reported for the entire study group and, in parentheses, for the subset of patients with regional wall motion abnormalities.

in modest but statistically significant improvement in the accuracy of interpretation from $77 \%$ to $81 \%$, as well as sensitivity (from $80 \%$ to $83 \%$ ) and specificity (from $76 \%$ to $80 \%$ ). In addition, the interobserver variability was markedly reduced from $52 \%$ to $33 \%$ by combined reading of dynamic and still-frame parametric images.

In the subset of patients with regional wall motion abnormalities, the accuracy of interpretation based on dynamic MR images was lower (71\%) and the interobserver variability higher $(58 \%)$ than in the entire study population. The improvements in the calculated accuracy and interobserver variability achieved by adding parametric images were similar in this subset of patients to those noted in all 18 study subjects (Table 1).

\section{DISCUSSION}

Clinical assessment of LV wall motion is mostly based on visual interpretation of dynamic ultrasound and, more recently, MR images of the heart. This methodology heavily relies on the reader's ability to efficiently extract and integrate spatial and temporal information on endocardial motion and wall thickening, which requires extensive training and is known to be subjective and experience dependent (Picano et al., 1991; Popp et al., 1998; Stewart et al., 1995). Different approaches for quantitative evaluation of regional wall motion have been proposed in the past to overcome the subjective nature of the conventional echocardiographic interpretation (Assmann et al., 1993; Bates et al., 1994; Force et al., 1984; Ginzton et al., 1986; Haendchen et al., 1983; Klausner et al., 1982; Moynihan et al., 1981; Pandian et al., 1983; Parisi et al., 1981; Schnittger et al., 1984; Sheehan et al., 1986). However, none of these techniques is widely used in routine clinical practice because they are based on extensive analysis of multiple consecutive frames.
We recently developed a quick and automated technique for still-frame parametric imaging of endocardial motion, which was favorably tested on echocardiographic images (Caiani et al., 2002). We used parametric images of the amplitude of the local oscillations of videointensity throughout the cardiac cycle as a basis for a simple display of endocardial motion in which the spatial and temporal information is naturally integrated. We found that this display, when combined with dynamic images, improved the accuracy and reduced the interobserver variability of the interpretation of LV wall motion made by relatively inexperienced readers of echocardiographic images. Our method was not intended to replace the conventional visual interpretation, but rather supplement it, because the ability of the trained human brain to integrate spatial and temporal information and take into account the limitations and distortions due to technical abnormalities is very difficult to substitute with an automated computerized technique.

Although echocardiographic images are usually acquired in dedicated cardiac imaging laboratories and interpreted by cardiologists trained in the evaluation of LV wall motion, cardiac MR imaging is commonly performed in radiology departments, and images are reviewed by physicians without dedicated training and extensive experience with this methodology. As a result, the detection of regional wall motion abnormalities from MR images may be less accurate and may suffer from high interobserver variability. In the present study, we tested the hypothesis that, similar to echocardiography, adding computer-generated stillframe parametric images of $\mathrm{LV}$ wall motion to the dynamic loops of cardiac MR images could improve the accuracy and reduce the interobserver variability of the assessment of LV wall motion by non-cardiologists who occasionally interpret cardiac MR images for regional $\mathrm{LV}$ function.

To the best of our knowledge, still-frame parametric imaging of $\mathrm{LV}$ wall motion has not been 
described previously. We chose the amplitude of local videointensity oscillations as the parameter to be displayed based on the hypothesis that pixel transitions between blood and tissue would generate strong periodic changes in videointensity in areas spanned by the endocardial motion over the cardiac cycle. Because of the high contrast between the LV cavity and the surrounding myocardium in the MR images, we expected these changes to be significantly lower in other portions of the image. Not only our results confirmed this hypothesis but we also found that parametric images maintained the morphologic features of the heart, thus allowing the reader to relate this information to the underlying cardiac structures, and in particular, to easily identify the location of the detected wall motion abnormalities.

Our choice of the sum of amplitudes of the first two harmonics of the variations in local videointensity, rather than the first harmonic alone, to represent wall motion in the parametric images was an improvement over the technique previously tested with echocardiographic images. This improvement was introduced to reduce artifacts we have seen in patients with abnormal septal motion due to conduction abnormalities and right ventricular volume overload. Because this abnormal septal motion is typically biphasic, the incorporation of the higher harmonic frequency was found to reduce the intensity dropout, which could otherwise be misidentified as hypokinesis.

Our results showed that the accuracy of the interpretation of regional LV wall motion by noncardiologists could be improved by adding parametric images of wall motion, which can be obtained by a quick and fully automated procedure. We also demonstrated the benefits of using this technique in patients with regional wall motion abnormalities, where accurate interpretation may require even higher levels of expertise than in subjects with normal wall motion. Importantly, the dramatic reduction in the interobserver variability of the non-expert interpretation indicates that this technique may potentially become a useful aid for the less experienced reviewers.

Nevertheless, it is important to remember that the main limitation of this technique is its high sensitivity to noise that may be caused by cardiac translation. Another limitation is that the presence of the papillary muscles or chordae tendinae in the imaging plane during part of the cardiac cycle may affect the parametric images and confound the interpretation of wall motion. However, these artifacts in the parametric images can be easily identified, because both translation and the presence of the papillary muscles are easily detectable in dynamic MR images, which are reviewed in conjunction with the parametric images. An additional limitation of this feasibility study is the small number of patients. This approach needs to be tested in the future in a larger group of patients. Also, in this pilot study, regional wall motion was classified as normal or abnormal, without differentiating the degree of abnormality. The impact of parametric images on grading wall motion abnormalities, as well as their use in specific disorders such as hypertrophic cardiomyopathy, have yet to be determined.

In summary, the results of this study proved the feasibility of still-frame parametric imaging of endocardial motion based on cardiac MR imaging. These images provide additional information that improves the accuracy and reduces the interobserver variability of the detection of regional wall motion abnormalities by non-cardiologists who are required to interpret cardiac images.

\section{ACKNOWLEDGMENTS}

We thank Drs. Steven Zangan and Anupinder Hazra for the time and effort they spent reviewing the images for this study.

\section{REFERENCES}

Assmann, P., Slager, C., van der Borden, S., Tijssen, J., Oomen, J., Roelandt, J. (1993). Comparison of models for quantitative left ventricular wall motion analysis from two-dimensional echocardiograms during acute myocardial infarction. Am. J. Cardiol. 71:1262-1269.

Bates, J., Ryan, T., Rimmerman, C., Segar, D., Sawada, S., Fitch, G., Feigenbaum, H. (1994). Color coding of digitized echocardiograms: description of a new technique and application in detecting and correcting for cardiac translation. J. Am. Soc. Echocardiogr. 7:363-369.

Caiani, E. G., Lang, R. M., Korcarz, C. E., DeCara, J. M., Weinert, L., Collins, K. A., Spencer, K. T., Mor-Avi, V. (2002). Improvement in echocardiographic evaluation of left ventricular wall motion using still-frame parametric imaging. J. Am. Soc. Echocardiogr. 15(9):926-934.

Cohen, J. L., Cheirif, J., Segar, D., Gillam, L. D., Gottdiener, J. S., Hausnerova, E., Bruns, D. E. (1998). Improved left ventricular endocardial border delineation and opacification with Optison 
(FS069), a new echocardiographic contrast agent-results of a phase III multicenter trial. $J$. Am. Coll. Cardiol. 32:746-752.

Force, T., Bloomfield, P., O’Boyle, J., Khuri, S., Josa, M., Parisi, A. (1984). Quantitative two-dimensional echocardiographic analysis of regional wall motion in patients with perioperative myocardial infarction. Circulation 70(2):233-241.

Ginzton, L., Conant, R., Brizendine, M., Thigpen, T., Laks, M. (1986). Quantitative analysis of segmental wall motion during maximal upright dynamic exercise: variability in normal adults. Circulation 73(2):268-275.

Haendchen, R., Wyatt, H., Maurer, G., Zwehl, W., Bear, M., Meerbaum, S., Corday, E. (1983). Quantitation of regional cardiac function by twodimensional echocardiography I. Patterns of contraction in the normal left ventricle. Circulation 67(6): $1234-1245$.

Klausner, S., Blair, T., Bulawa, W., Jeppson, G., Jensen, R., Clayton, P. (1982). Quantitative analysis of segmental wall motion throughout systole and diastole in the normal human left ventricle. Circulation 65(3):580-590.

Moynihan, P., Parisi, A., Feldman, C. L. (1981). Quantitative detection of regional left ventricular contraction abnormalities by two-dimensional echocardiography I. Analysis of methods. Circulation 63(4):752-760.

Pandian, N., Skorton, D., Collins, S., Falsetti, H., Burke, E., Kerber, R. (1983). Heterogeneity of left ventricular segmental wall thickening and excursion in 2-dimensional echocardiograms of normal human subjects. Am. J. Cardiol. 51:1667-1673.

Parisi, A. F., Moynihan, P. F., Folland, E. D., Feldman, C. L. (1981). Quantitative detection of regional left ventricular contraction abnormalities by two- dimensional echocardiography II. Accuracy in coronary artery disease. Circulation 4:761-767.

Picano, E., Lattanzi, F., Orlandini, A., Marini, C., L'Abbate, A. (1991). Stress echocardiography and the human factor: the importance of being expert. J. Am. Coll. Cardiol. 17(3):666-669.

Popp, R. L., Agatston, A., Armstrong, W. F., Nanda, N. C., Pearlman, A., Rakowski, Seward, J. B., Silverman, N. H., Smith M., Stewart, W. J., Taylor, R., Thys, D., Davis, C. (1998). Recommendations for training in performance and interpretation of stress echocardiography. J. Am. Soc. Echocardiogr. 11:95-96.

Schiller, N. B., Shah, P. M., Crawford, M., DeMaria, A., Devereux, R., Feigenbaum, H., Gutgessel, H., Reichek, N., Sahn, D., Schnittger, I., Silverman, N. H., Tajik, A. J. (1989). Recommendations for quantitation of the left ventricle by two-dimensional echocardiography. J. Am. Soc. Echocardiogr. 2:358-367.

Schnittger, I., Fitzgerald, P. J., Gordon, E. P., Alderman, E. L., Popp, R. L. (1984). Computerized quantitative analysis of left ventricular wall motion by two-dimensional echocardiography. Circulation $70(2): 242-254$.

Sheehan, F. H., Bolson, E. L., Dodge, H. T., Mathey, D. G., Schofer, J., Woo, H. W. (1986). Advantages and applications of the centerline method for characterizing regional ventricular function. Circulation 74(2):293-305.

Stewart, W. J., Aurigemma, G. P., Bierman, F. Z., Gardin, J. M., Kisslo, J. A. Jr., Pearlman, A. S., Seward, J. B., Weyman, A. E. (1995). Guidelines for training in adult cardiovascular medicine. Core Cardiology Training Symposium (COCATS). Task Force 4: training in echocardiography. $J$. Am. Coll. Cardiol. 25(1):16-19. 


\section{Request Permission or Order Reprints Instantly!}

Interested in copying and sharing this article? In most cases, U.S. Copyright Law requires that you get permission from the article's rightsholder before using copyrighted content.

All information and materials found in this article, including but not limited to text, trademarks, patents, logos, graphics and images (the "Materials"), are the copyrighted works and other forms of intellectual property of Marcel Dekker, Inc., or its licensors. All rights not expressly granted are reserved.

Get permission to lawfully reproduce and distribute the Materials or order reprints quickly and painlessly. Simply click on the "Request Permission/ Order Reprints" link below and follow the instructions. Visit the U.S. Copyright Office for information on Fair Use limitations of U.S. copyright law. Please refer to The Association of American Publishers' (AAP) website for guidelines on Fair Use in the Classroom.

The Materials are for your personal use only and cannot be reformatted, reposted, resold or distributed by electronic means or otherwise without permission from Marcel Dekker, Inc. Marcel Dekker, Inc. grants you the limited right to display the Materials only on your personal computer or personal wireless device, and to copy and download single copies of such Materials provided that any copyright, trademark or other notice appearing on such Materials is also retained by, displayed, copied or downloaded as part of the Materials and is not removed or obscured, and provided you do not edit, modify, alter or enhance the Materials. Please refer to our Website User Agreement for more details.

\section{Request Permission/Order Reprints}

Reprints of this article can also be ordered at http://www.dekker.com/servlet/product/DOI/120038083 